\title{
Intestinal helminth infection impacts the systemic distribution and function of the naive lymphocyte pool
}

\author{
IL King ${ }^{1}, \mathrm{~K} \mathrm{Mohrs}^{2, \star}$, AP Meli ${ }^{1}$, J Downey ${ }^{3}, \mathrm{P}_{\text {Lanthier }}^{2}$, F Tzelepis ${ }^{4}$, JH Fritz ${ }^{5}$, AV Tumanov ${ }^{6}$, \\ M Divangahi ${ }^{3,4,7}$, EA Leadbetter ${ }^{6}$ and $M$ Mohrs ${ }^{2, *}$
}

Homeostasis is a fundamental principle of biological systems. A paradigm of immune homeostasis is the remarkably constant number of naive T and B lymphocytes in the body that continuously circulate through the secondary lymphoid organs to maximize immune surveillance. Whether the dynamics and distribution of the systemic naive lymphocyte pool is affected following organ-specific infection is not known. Here we show that, following infection of mice with an enteric helminth, naive $T$ and $B$ lymphocytes accumulate in the $T$ helper type 2-reactive mesenteric lymph node while they are concurrently depleted from non-draining peripheral lymph nodes. This systemic redistribution of naive lymphocytes is sustained into the chronic phase of the infection, requires lymphotoxin beta receptor-dependent signals and is associated with a reduced ability of parasitized animals to mount antigen-specific cellular and humoral immune responses to heterologous immunization or infection at peripheral sites. Our data suggest that the function of the homeostatic naive lymphocyte pool can be modulated by its systemic distribution following infection and may provide a novel concept underlying compromised immune responsiveness at peripheral sites in helminth-infected individuals.

\section{INTRODUCTION}

Continuous circulation of the homeostatic naive lymphocyte population through lymph nodes (LNs) is a central tenet of immune surveillance. Cellular entry into and exit from these LNs are at equilibrium in the absence of overt infection or inflammation such that the number of lymphocytes present in a given LN remains remarkably constant during adult life. ${ }^{1}$ One possible reason that this set point evolved is to optimize the environment for lymphocyte survival as well as activation upon specific antigen encounter. Indeed, circulation through LNs provides access to limited survival signals such as interleukin (IL)-7 that are required for cell survival. ${ }^{2}$ In further support of this hypothesis are studies in which naive $\mathrm{T}$ cells of known specificity were transferred at carefully titrated numbers into recipient animals prior to peptide immunization. ${ }^{3}$ This work demonstrated that, when the number of transferred cells exceeds physiological levels, the proliferative capacity of the donor cells was compromised. Thus LN cell number must be precisely controlled to maintain immune homeostasis and maximize responsiveness upon immune challenge.

Although homeostatic control of T- and B-cell populations in the LNs is well established, the impact that local lymphoid tissue inflammation may have on the systemic pool of lymphocytes is less clear. One consequence of infection or immunization-induced inflammation is transient enlargement of LNs draining the site of immune challenge. Factors contributing to LN hyperplasia include expansion of the lymphatic, ${ }^{4}$ fibroblast reticular cell, ${ }^{5}$ and arterial networks ${ }^{6,7}$ that collectively enhance leukocyte entry into the draining $\mathrm{LN}$ as well as proliferative effector cell expansion and retention. ${ }^{8}$

${ }^{1}$ Department of Microbiology and Immunology, Microbiome and Disease Tolerance Centre, McGill University, Montreal, Quebec, Canada. ${ }^{2}$ Trudeau Institute, Saranac Lake, New York, USA. ${ }^{3}$ Department of Pathology, Meakins-Christie Laboratories, McGill University Health Centre, Montreal, Quebec, Canada. ${ }^{4}$ Department of Medicine, MeakinsChristie Laboratories, McGill International TB Centre, McGill University Health Centre, Montreal, Quebec, Canada. ${ }^{5}$ Department of Microbiology and Immunology, Department of Physiology, Complex Traits Group, McGill University, Montreal, Quebec, Canada. ${ }^{6}$ Department of Microbiology and Immunology, University of Texas School of Medicine Health Science Center at San Antonio, San Antonio, Texas, USA and ${ }^{7}$ Department of Microbiology and Immunology, McGill University, Montreal, Quebec, Canada. Correspondence: IL King or M Mohrs (irah.king@mcgill.ca or markus.mohrs@regeneron.com)

*Present address: Regeneron Pharmaceuticals, Inc., Tarrytown, New York, USA. 
In experiments examining the primary $\mathrm{T}$ helper type 2 (Th2)driven immune response to Heligmosomoides polygrus $(H p)$, a strictly enteric helminth parasite, we observed dramatic and sustained enlargement of the mesenteric lymph nodes (mesLNs), the primary site of adaptive immune cell activation following infection. In addition to robust expansion of $\mathrm{CD} 4^{+} \mathrm{T}$ and $\mathrm{B}$ effector cells, we unexpectedly observed a significant and persistent accumulation of naive $\mathrm{T}$ and $\mathrm{B}$ cells in the inflamed mesLNs. Subsequent experiments were performed to understand (1) the relevance of naive lymphocyte accumulation to the primary effector cell response, (2) the mechanisms underlying this accumulation, and (3) its impact on the systemic distribution of the naive lymphocyte pool and response to secondary challenge.

\section{RESULTS}

Naive lymphocytes accumulate in the Th2-reactive mesLNs following enteric helminth infection

Infection of mice with the enteric helminth parasite $H p$ results in a robust Th2 and type 2 humoral response initiated in the mesLNs. ${ }^{9,10}$ This response results in a striking enlargement of the mesLNs by 2 weeks postinfection and a dramatic increase in mesLN weight and total lymphocyte number (hyperplasia) as well as morphological changes owing to a robust humoral immune response (Figure 1a-c). Hp-induced mesLN hyperplasia persisted through the chronic stage of infection (i.e., 6 weeks), yet was reduced if the parasite was eliminated using anti-helminthics at 2 weeks postinfection (Figure 1c,d). Further characterization of lymphocyte subsets in the Th2-reactive mesLNs over the course of infection indicated that the total number of $\mathrm{CD} 4^{+}$and $\mathrm{CD} 8^{+} \mathrm{T}$ cells significantly increased between 1 and 4 weeks postinfection with the $\mathrm{CD} 19^{+}$ B-cell pool increasing with slightly more rapid kinetics (Figure 1e). In addition to an expected increase in the number of $\mathrm{CD} 44^{\text {hi }} \mathrm{CD} 62 \mathrm{~L}^{\text {lo }}$ effector lymphocytes (Figure 1f, see Supplementary Figure S1a-c online for gating), we also noticed an unexpected increase in the number of $\mathrm{CD} 44^{\mathrm{lo}} \mathrm{CD} 62 \mathrm{~L}^{\mathrm{hi}}$-naive phenotype lymphocytes. In fact, the majority of the $\mathrm{T}$ and $\mathrm{B}$ lymphocytes present in the inflamed mesLNs expressed a naive phenotype (Figure 1g). These results demonstrate that naive $\mathrm{T}$ - and B-cell accumulation occurs within the Th2-reactive LN following helminth infection.

\section{The accumulation of naive lymphocytes is driven by an increased homeostatic set point}

Th2 differentiation and IL-4 production is critical for protective immunity to $H p$ infection. ${ }^{11}$ IL-4 cytokine reporter (4get) mice, in which green fluorescent protein (GFP) identifies IL-4expressing cells, are a sensitive ex vivo readout for Th2 differentiation. ${ }^{12}$ We have previously shown that the number of IL-4/GFP ${ }^{+}$effector CD4 T cells in the mesLNs begins to increase within the first 5 days of $H p$ infection and continues to expand over time, reaching its peak between 2 and 4 weeks postinfection. ${ }^{13}$ In contrast, naive $\mathrm{T}$ cells began to accumulate in the Th2-reactive mesLNs only after 7 days postinfection (Figure 1g). To determine whether this accumulation contributed to the IL- $4^{+}$effector T-cell response, 4get mice were treated with anti-CD62L monoclonal antibody between days 7 and 14 postinfection to inhibit naive lymphocyte entry into the mesLNs. ${ }^{14}$ Analysis on day 14 postinfection revealed a dramatic reduction in naive $\mathrm{T}$ - and $\mathrm{B}$-cell accumulation, as measured by a $\mathrm{GFP}^{-} \mathrm{CD} 44^{\mathrm{lo}}$ or $\mathrm{IgD}^{+} \mathrm{CD} 44^{\text {lo }}$ phenotype (in lieu of $\mathrm{CD} 62 \mathrm{~L}$ ), respectively, in the mesLNs of infected mice (Figure 2a); however, no significant defect in the number of IL- ${ }^{+}$effector CD4 $\mathrm{T}$ cells was observed (Figure 2b). To further determine whether the period between 1 and 2 weeks postinfection represented a critical phase for naive $\mathrm{T}$ - and $\mathrm{B}$-cell accumulation, some mice treated with anti-CD62L were examined 1 week after antibody treatment was terminated (i.e., day 21 postinfection). Release of the entry blockade after the second week of infection restored the number of naive $T$ and B cells to isotype-treated control levels. However, again we did not observe any difference in the number of IL- $4^{+}$effector CD4 $\mathrm{T}$ cells at this time point (Figure 2a,b). Collectively, these data indicate that the accumulation of naive $\mathrm{T}$ cells in the reactive mesLNs beginning 1 week after infection does not affect the IL-4 ${ }^{+}$effector CD4 T-cell response and that the reactive mesLNs represents a sustained reservoir for naive lymphocytes owing to an increased homeostatic set point.

\section{$\mathrm{CD}^{+} \mathrm{T}$-cell activation is required for naive $\mathrm{B}$ lymphocyte accumulation}

A previous study using subcutaneous immunization with antigen in Th1-polarizing CFA (complete Freund's adjuvant) demonstrated that B cells were important for LN enlargement. ${ }^{4}$ To determine whether $\mathrm{B}$ cells also contribute to naive $\mathrm{T}$-cell accumulation in a Th2-dominated immune response, BALB/c or B-cell-deficient JhD mice were infected with $H p$ and naive $\mathrm{T}$ cells were enumerated in the mesLNs 2 weeks later. In contrast to CFA immunization, ${ }^{4}$ the number of naive $\mathrm{T}$ cells was not decreased in the absence of B cells but in fact increased compared with WT controls, suggesting a potential regulatory role for B lymphocytes in naive T-cell accumulation (Figure 3a). Using a model of viral infection, Iwasaki and colleagues showed that innate immune mechanisms contributed to transient LN enlargement and naive T-cell accumulation within $48 \mathrm{~h}$ of immune challenge. ${ }^{6}$ In the case of $H p$ infection, however, we did not observe naive $\mathrm{T}$ lymphocyte accumulation prior to day 7 postinfection (Figure 1a), suggesting that the adaptive immune response may have a more dominant role. Using $\mathrm{CD} 19^{+} \mathrm{IgD}{ }^{+} \mathrm{CD} 44^{\text {lo }} \mathrm{B}$ cells as a readout for naive lymphocyte accumulation, $\mathrm{BALB} / \mathrm{c}, \mathrm{TCR} \alpha$-deficient $\mathrm{BALB} / \mathrm{c}$, and $\mathrm{CD} 4$ T-cell-depleted $\mathrm{BALB} / \mathrm{c}$ mice were infected with $H p$ and the number of naive $B$ cells was determined 2 weeks later. In contrast to B-cell-deficient mice, infected animals that lack $\alpha \beta$ $\mathrm{T}$ cells or were depleted of $\mathrm{CD} 4^{+}$cells failed to accumulate naive $B$ cells in their draining mesLNs (Figure $\mathbf{3 b}, \mathbf{c}$ ). mesLNs from $H p$-infected DO11.10 mice containing a monoclonal population of $\mathrm{CD} 4^{+} \mathrm{T}$ cells specific for chicken ovalbumin (OVA) also failed to accumulate naive B cells (Figure 3d), indicating that not just $\mathrm{CD} 4^{+} \mathrm{T}$ cells per se but activation of this population by cognate antigen was required. 
a

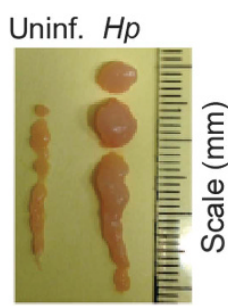

b

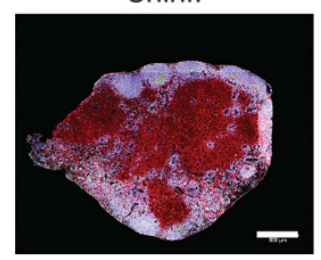

Hp 2 wk

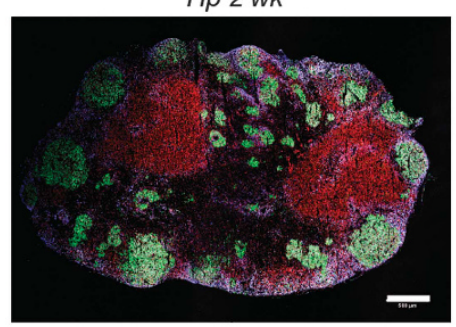

d

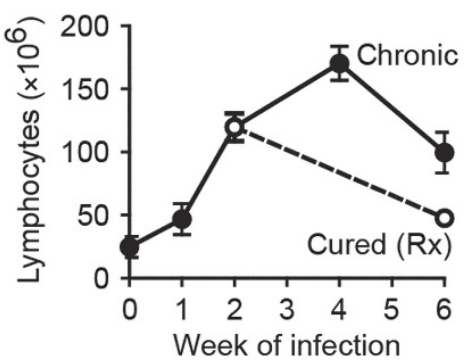

c

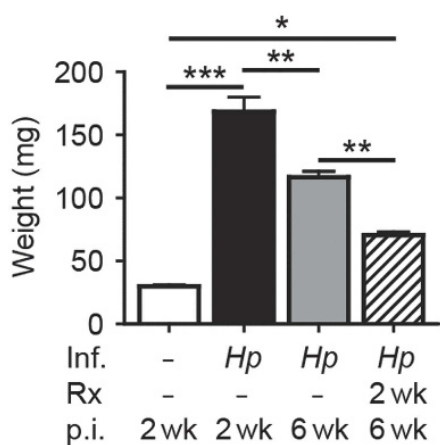

g
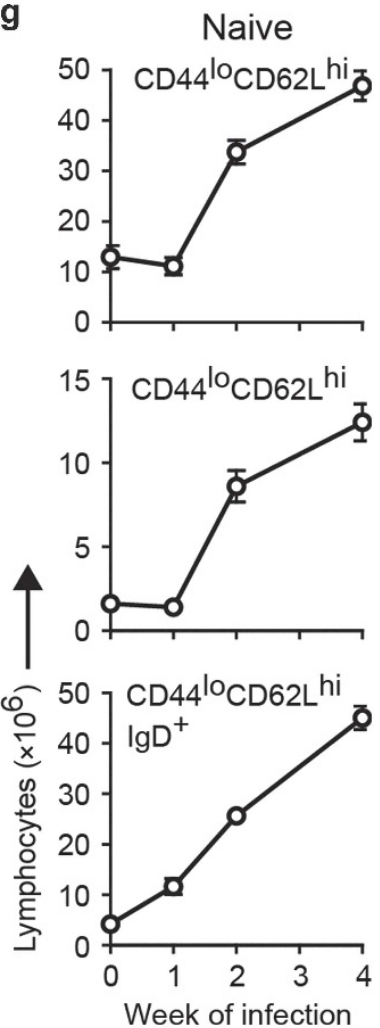

Figure 1 Naive lymphocytes accumulate in the T helper type 2-reactive mesenteric lymph nodes (mesLNs) following intestinal helminth infection. $\mathrm{BALB} / \mathrm{c}$ mice were infected with Heligmosomoides polygrus $(H p)$ or remained naive and the mesLNs were collected at the indicated time points. (a) Representative image of mesLNs from naive and 2 week $\mathrm{Hp}$-infected animals. (b) Confocal immunofluorescent images of uninfected and 2-week $\mathrm{Hp}$ infected mesLNs. B220, white; CD4, red; GL7, green. Bar, $500 \mu \mathrm{m}$. (c) mesLN weight from naive and Hp-infected animals. Some mice received antihelminthics at 2 weeks post-Hp infection; p.i., postinfection. (d) Number of total or (e) $\mathrm{CD}^{+}, \mathrm{CD}^{+}$, and CD19 ${ }^{+}$lymphocytes in the mesLNs at the indicated times after infection. (f) Number of effector and (g) naive phenotype CD4 ${ }^{+}, \mathrm{CD} 8^{+}$, and CD19 ${ }^{+}$lymphocytes at the indicated time points after infection. Each data point shows the mean of $4-5$ individual mice and error bars indicate the s.e.m. Data are representative of at least three independent experiments. ${ }^{\star} P<0.05,{ }^{* *} P<0.01,{ }^{\star * *} P<0.001$. 


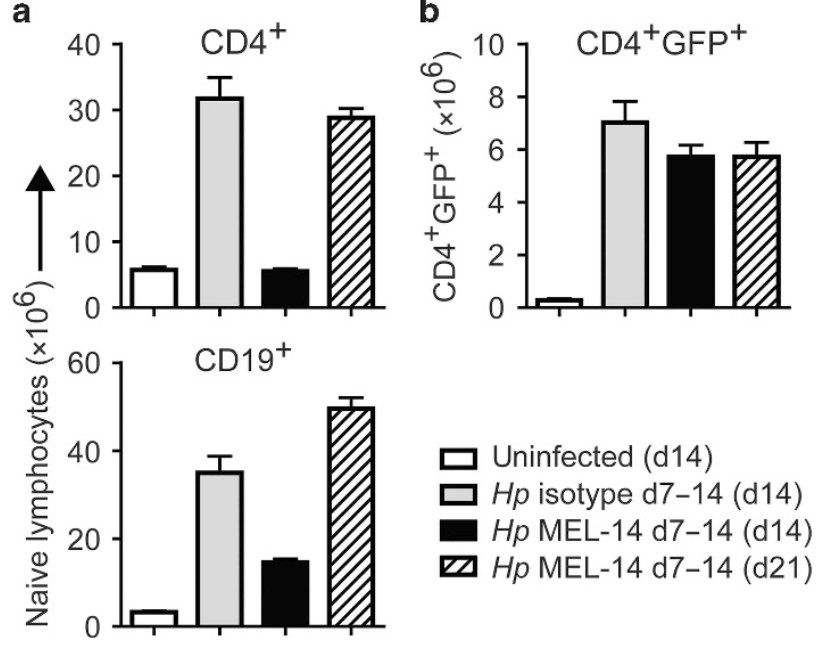

Figure 2 Delayed naive lymphocyte accumulation is dispensable for interleukin (IL)- $4^{+}$effector CD4 T-cell differentiation. BALB/c 4get mice were infected with Heligmosomoides polygrus $(H p)$ or remained uninfected and were treated every other day with anti-CD62L (MEL-14) or rlgG2a isotype control during the indicated period. Mesenteric lymph nodes (mesLNs) were analyzed on day 14 or 21 postinfection as specified in parentheses. (a) Number of naive phenotype CD4 ${ }^{+}$and $\mathrm{CD} 19^{+}$ lymphocytes. (b) Number of GFP ${ }^{+} \mathrm{IL}-4^{+}$effector CD4 T cells. Each data point shows the mean of $4-5$ individual mice and error bars indicate the s.e.m. Data are representative of two independent experiments.

\section{Lymphotoxin- $\beta$ receptor signaling is required for naive lymphocyte accumulation}

Because naive lymphocyte accumulation required $\mathrm{CD} 4{ }^{+} \mathrm{T}$-cell activation but was not required for the increase in IL- $4^{+}$ effector CD4 T cells after 1 week of infection, we sought to identify factors that regulated naive $\mathrm{T}$-cell accumulation independent of the Th2 response. These events could not be separated in mice unable to receive IL-4 signals because both naive T-cell accumulation and, consistent with our previous studies, ${ }^{15}$ the Th2 response was compromised following $H p$ infection of IL-4 R $\alpha$ knockout (KO) 4get mice (Figure 4a,b). In addition, experiments using mice deficient in tumor necrosis factor (TNF)- $\alpha$ or type I interferon receptor (IFNAR) signals, two pathways previously shown to promote LN hyperplasia, ${ }^{16,17}$ demonstrated that these factors were not only dispensable for naive lymphocyte accumulation following $H p$ infection but may also actually exert regulatory effects, at least regarding T-cell accumulation (Figure $\mathbf{4 c , d}$ and see Supplementary Figure S3a,b).

Lymphotoxin $\beta$ receptor (LT $\beta$ R) has been previously shown to have a role in type 2 immunity following $H p$ infection and mice deficient in the LT $\beta R$ ligands experience compromised LN hyperplasia following subcutaneous immunization with CFA. $^{18-20}$ However, in these studies LT $\beta R$ signals were inhibited at the time of infection or immunization, potentially compromising initial dendritic cell (DC)-dependent T-cell recruitment to the mesLNs. ${ }^{21}$ As naive T lymphocytes do not accumulate prior to establishment of a robust Th2 response (i.e., 1 week after $H p$ infection; Figure 1), we blocked LT signaling by administering LT $\beta$ R-Ig on days 7 and 10 and
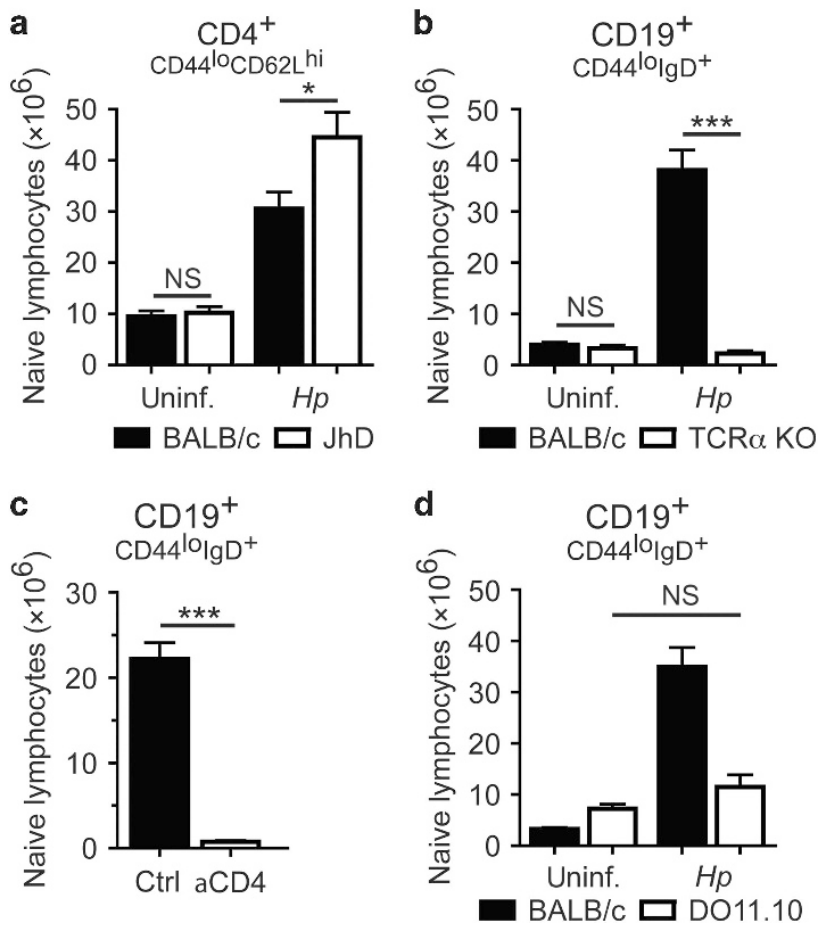

Figure $3 \mathrm{CD}^{+}$T-cell activation is required for naive $\mathrm{B}$ lymphocyte accumulation. (a) B-cell-deficient JhD mice and BALB/c wild-type (WT) controls were infected with Heligmosomoides polygrus $(\mathrm{Hp})$ or remained uninfected and the number of naive phenotype CD4 ${ }^{+} \mathrm{T}$ cells in the mesenteric lymph nodes (mesLNs) was analyzed 2 weeks later. (b) T-cell-deficient TCR $\alpha$ knockout (KO) mice and BALB/c WT controls were infected with $\mathrm{Hp}$ or remained naive and the number of naive phenotype $\mathrm{CD}_{19}{ }^{+} \mathrm{B}$ cells in the mesLNs was analyzed 2 weeks later. (c) BALB/c mice were treated with anti-CD4 or rlgG2b isotype control and infected with $\mathrm{Hp} 1$ day later. The number of naive phenotype CD19 ${ }^{+} \mathrm{B}$ cells in the mesLNs was determined at 2 weeks postinfection. (d) DO11.10 TCR transgenic mice and BALB/c WT controls were infected with $H p$ and the number of naive phenotype $C D 19^{+} B$ cells in the mesLNs was determined at 2 weeks post-infection. Each data point shows the mean of 3-5 individual mice and error bars indicate the s.e.m. Data are representative of at least two independent experiments. NS, not significant; ${ }^{*} P<0.05$, ${ }^{* * *} P<0.001$

assessed naive T-cell accumulation and the IL- $4^{+}$effector CD4 T-cell response on day 14. Compared with control animals, naive $\mathrm{T}$ and $\mathrm{B}$ cells failed to accumulate in the mesLNs of LT $\beta R$-Ig-treated mice (Figure 4e). Importantly, the treatment resulted only in a subtle, albeit significant, reduction in the number of IL- $4^{+}$expressing CD ${ }^{+}$T cells (Figure 4f). These results were most likely not due to a change in lymphocyte survival as the frequency of active Caspase- $3^{+}$cells (a marker of apoptosis) was not different between the groups (see Supplementary Figure S3c-e).

A previous report described an important role for LT $\beta$ production by B cells in LN hyperplasia and remodeling during $H p$ infection. ${ }^{20}$ Although we found that LT signals were also important for naive T-cell accumulation, our results indicated that $\mathrm{B}$ cells were not required suggesting that other cellular sources of LT $\beta R$ ligands contribute to this process. To examine whether other LN cell populations express ligands for LT $\beta$, including LT $\alpha \beta$ and LIGHT, DCs, B cells, and CD4 ${ }^{+}$T cells were sorted from the mesLNs of $H p$-infected mice and Lta, Ltb, 

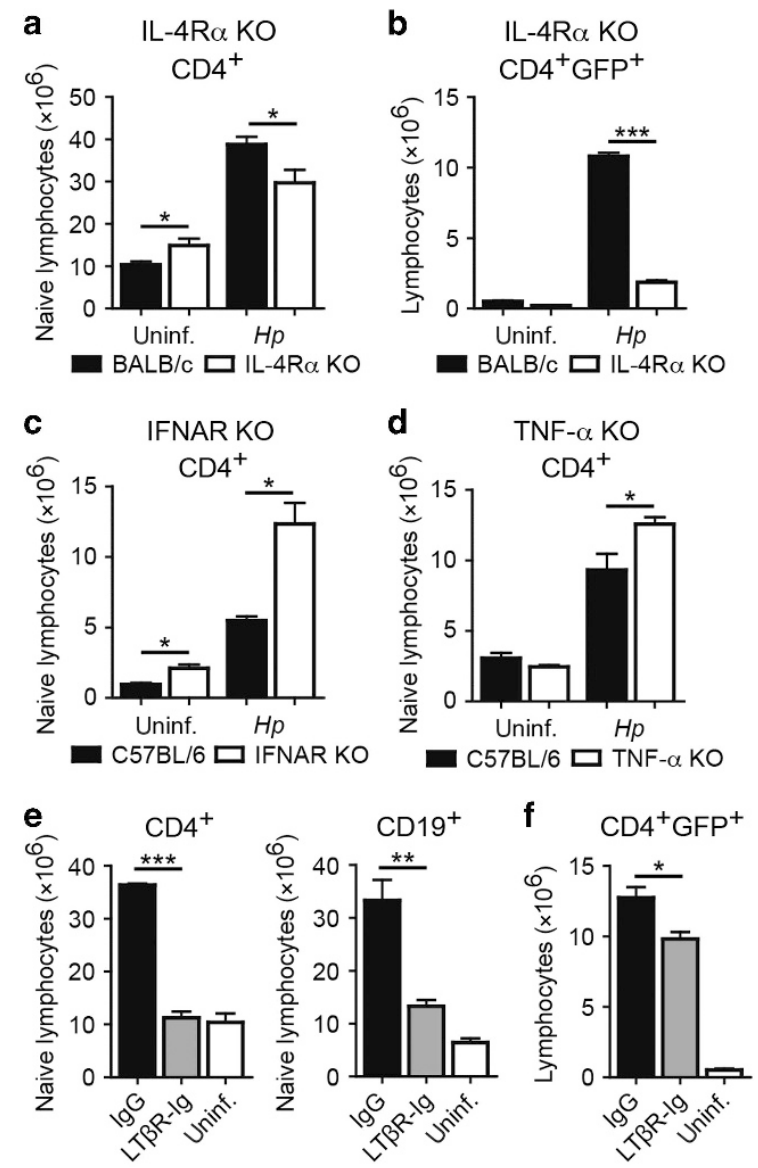

g

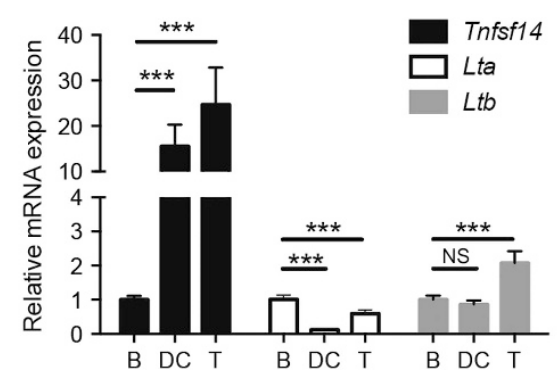

Figure 4 Lymphotoxin- $\beta$ receptor signaling is required for naive lymphocyte accumulation. (a, b) Interleukin (IL)-4R $\alpha$ deficient and wildtype (WT) BALB/c 4get mice were infected with Heligmosomoides polygrus $(H p)$ or remained naive and the number of naive (a) or IL-4/GFP ${ }^{+}$ effector (b) CD4 ${ }^{+} \mathrm{T}$ cells in the mesenteric lymph nodes (mesLNs) was analyzed at 2 weeks postinfection. IFNAR knockout (KO) mice (c) or tumor necrosis factor (TNF)- $\alpha$ KO mice (d) as well as C57BL/6 WT controls were infected with $\mathrm{Hp}$ or remained naive. The number of naive phenotype CD4 ${ }^{+}$ T cells in the mesLNs was determined 2 weeks later. (e, f) BALB/c 4 get mice were infected with $H p$ and LT $\beta$ R-Ig or control IgG was administered intraperitoneally on days 7 and 10 after infection. The number of naive $\mathrm{CD}^{+}{ }^{+} \mathrm{T}$ and $\mathrm{CD} 19^{+} \mathrm{B}$ cells (e) or effector phenotype CD4 ${ }^{+} \mathrm{T}$ cells $(\mathbf{f})$ in the mesLNs was analyzed at 2 weeks postinfection. (g) $\mathrm{B} 220^{+} \mathrm{CD} 4^{-} \mathrm{B}$ cells, $\mathrm{CD} 11 \mathrm{c}^{+} \mathrm{MHClI}^{+}$, and $\mathrm{CD} 4^{+} \mathrm{B}_{220^{-}} \mathrm{T}$ cells were sorted from the mesLNs of 2 week $\mathrm{Hp}$-infected mice and mRNA from each population was assessed by quantitative reverse transcriptase-PCR for the indicated transcripts. Each group shows the mean of eight individual mice and error bars indicate the s.e.m. Data are representative of at least two independent experiments. NS, not significant; ${ }^{\star} P<0.05$, ${ }^{\star \star} P<0.01$ ${ }^{* * *} P<0.001$. and Tnfsf14 (encoding for LIGHT) were measured by quantitative reverse transcriptase-PCR. Although both Lta and $L t b$ were expressed at high levels by all immune cell subsets examined (data not shown), their relative difference compared with B cells was small (Figure $\mathbf{4 g}$ ). By contrast, both DCs and $\mathrm{CD} 4^{+} \mathrm{T}$ cells expressed high levels of Tnfsf 14 mRNA compared with B cells (Figure 4g). Collectively, these data indicate that LT $\beta R$-dependent signals, possibly from multiple immune cell subsets, orchestrate the accumulation of naive lymphocytes in the inflamed mesLNs independent of its effects on initial Th2 differentiation or cell survival following helminth infection.

\section{Loss of naive lymphocytes from non-draining LNs during Hp infection}

The striking and persistent accumulation of naive lymphocytes in the mesLNs of $H p$-infected animals prompted us to identify the site(s) from where these cells may derive. Although the thymus generates new naive $T$ lymphocytes and the spleen contains a large number of naive $\mathrm{T}$ and $\mathrm{B}$ cells that could potentially be recruited to inflamed sites, neither thymectomy nor splenectomy of adult mice prior to infection affected the accumulation of naive $\mathrm{T}$ and $\mathrm{B}$ cells (unpublished observations). However, the number of lymphocytes and naive $\mathrm{T}$ - and B-cell subsets in non-draining LNs (e.g., inguinal, axillary, and brachial LNs) were significantly reduced at 2 weeks after $H p$ infection compared with uninfected controls (Figure 5a). To test whether the naive lymphocyte redistribution occurred in an antigen-independent manner, OVA-specific $\mathrm{CD}^{+}{ }^{+} \mathrm{T}$ cells were purified from DO11.10 mice and transferred into Thyl congenic uninfected mice or mice infected with $H p 11$ days prior. One hour after transfer, 10-fold more donor KJ1-26 DO11.10 T cells were found in the mesLNs of $H p$-infected mice compared with uninfected controls (as determine by fluorescence-activated cell sorting (FACS); Figure 5b). Consistent with the infection-induced redistribution of the polyclonal naive lymphocyte population from the peripheral LNs, there were significantly fewer donor DO11.10 cells in the peripheral LNs of infected mice compared with uninfected controls (Figure $\mathbf{5 b}$ ). The full extent of the systemic redistribution was revealed by comparing the accumulation of donor $\mathrm{T}$ cells in the mesLNs relative to the skin-draining LNs in the same animal (Figure 5c). These data indicate that the systemic naive lymphocyte pool can be redistributed in an antigenindependent manner following an enteric helminth infection.

\section{$H p$ infection compromises immunity to heterologous challenge at peripheral sites}

Landmark studies by Moon et al. ${ }^{22}$ demonstrated that the starting number of naive lymphocytes specific for a given antigen predicts the magnitude of the primary adaptive immune response following immunization with relevant peptide. Because helminth infection effectively decreased the naive lymphocyte number in non-draining LNs, we reasoned that the magnitude of the immune response to heterologous challenge at peripheral sites might be compromised in helminth-infected mice. To test this hypothesis, we 

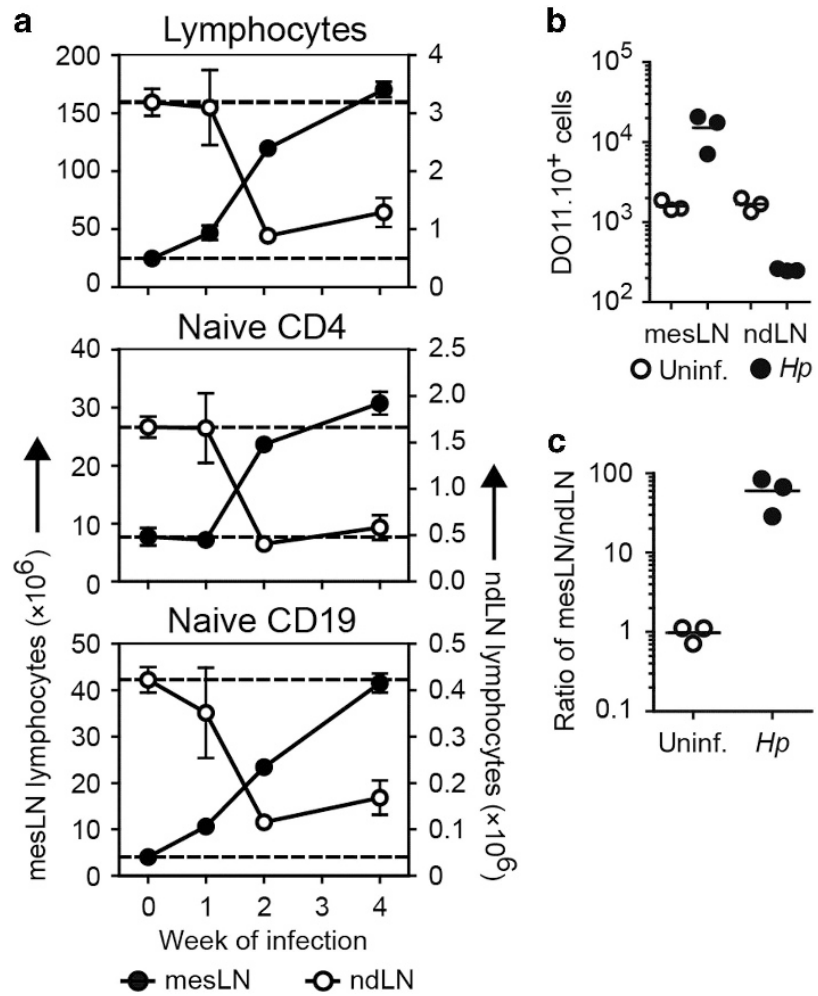

Figure 5 Intestinal helminth infection results in the systemic and sustained redistribution of the naive lymphocyte pool. (a) BALB/c or 4 get $\mathrm{BALB} / \mathrm{c}$ mice were infected with Heligmosomoides polygrus $(\mathrm{Hp})$ and the number of total lymphocytes and naive phenotype CD4 ${ }^{+} \mathrm{T}$ and $\mathrm{CD} 19^{+} \mathrm{B}$ cells in the mesenteric lymph nodes (mesLNs; left $y$ axis) and the pooled non-draining inguinal, axillary, and brachial LNs (ndLNs) (right yaxis) were determined at the indicated time points. Each data point shows the mean of 3-5 individual mice and error bars indicate the s.e.m.. Data are representative of at least two independent experiments. (b) Ovalbumin-

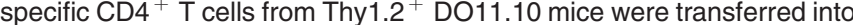
uninfected or day $11 \mathrm{Hp}$-infected Thy $1.1^{+}$congenic mice. The number of Thy $1.2^{+} \mathrm{KJ} 1-26^{+}$donor $\mathrm{CD} 4{ }^{+} \mathrm{T}$ cells in the mesLNs or the pooled ndLNs was determined $1 \mathrm{~h}$ later by flow cytometry. (c) The relative recruitment of DO11.10 donor cells into the mesLNs relative to the ndLNs was calculated based on the total numbers determined in (b). Scatter plots in (b) and (c) represent individual mice. Data are representative of two independent experiments with three mice per group.

employed several distinct methods of heterologous challenge to measure the antigen-specific response at peripheral sites of helminth-infected mice. First, at 2 weeks postinfection with $H p$, mice were immunized with the T-dependent antigen 4-hydroxy-3-nitrophenylacetyl (NP)-Keyhole limpet hemocyanin in alum, and 10 days after immunization, the frequency and number of NP-specific B cells in the skin-draining LNs was determined. Comparing immunized mice with or without concurrent $H p$ infection, the frequency of class-switched $\mathrm{IgD}^{-}$NP-specific B cells was similar between groups suggesting that activation of antigen-specific $B$ cells on a per cell basis was not compromised (Figure 6a and see Supplementary Figure S2). In contrast, the absolute number of NP-specific B cells in immunized mice infected with $\mathrm{H} p$ was significantly decreased compared with uninfected controls (Figure 6b). Concomitantly, serum titers of NP-specific IgG were decreased in helminth-infected mice compared with helminth-naive animals (Figure 6c). Similar differences in antigen-specific IgG titers were obtained using Prevnar13, a clinically relevant pneumococcal vaccine formulation (Figure 6d). To examine whether helminth infection also compromised immunity to heterologous infection at a distinct tissue site, $H p$-infected or naive mice were infected with influenza A virus. ${ }^{23}$ Seven days after influenza A virus infection (day-21 post- $H p$ infection), the lung-draining mediastinal LNs were visibly smaller in helminth-infected mice compared with controls (unpublished observations) and this corresponded with a lower number of total lymphocytes (Figure 6e). In addition, the number of influenza $A$ virus-specific $\mathrm{CD} 8{ }^{+} \mathrm{T}$ effector cells in the mediastinal LNs was decreased in helminth-infected mice and was associated with increased viral burden in lungs compared with control animals (Figure 6f,g). Collectively, these results suggest that helminth infection-induced systemic redistribution of naive lymphocytes compromises the magnitude of the immune response to heterologous immune challenge and reduced pathogen clearance at distinct barrier sites.

\section{DISCUSSION}

Helminth infections almost universally elicit Th2 responses that limit worm burden, promote tissue repair, and, in some cases, confer protection from re-infection. ${ }^{9}$ However, an excessive Th2 response can be detrimental to the host leading to immunopathology ${ }^{24}$ and reduced fitness against other infectious agents. ${ }^{25-27}$ Our results reveal another previously unknown consequence of enteric helminth infection, namely, the systemic and sustained redistribution of the naive lymphocyte pool away from peripheral LNs into the mesLNs resulting in compromised immune responses to heterologous peripheral challenge and increased susceptibility to infection. Although helminths including $H p$ produce immunosuppressive factors, ${ }^{28}$ we did not find a change in the frequency of the antigen-specific response in the LNs draining peripheral sites of immunized mice previously infected with $\mathrm{H} p$ compared with uninfected controls, suggesting a largely unimpeded priming of the heterologous immune response. However, we did observe a significant decrease in the magnitude of $\mathrm{T}$ and $\mathrm{B}$ effector cell response in three separate antigen-specific models of heterologous challenge. These data argue against direct immunosuppression by helminth-derived factors and instead suggests that an accumulation of naive lymphocytes in the reactive mesLNs compromised the availability of lymphocytes in peripheral LNs and subsequent antigen encounter and activation. Surprisingly, the redistribution and accumulation of naive lymphocytes in the mesLNs of helminth-infected animals was not required to support the effector T-cell response. The beneficial function that naive $\mathrm{T}$ lymphocyte accumulation may have on host immunity to helminth infection has yet to be determined. It is tempting to speculate that the increased number of naive $\mathrm{T}$ cells in the mesLNs enhances the ability to respond to additional local immune challenges that might be brought about by the compromised barrier integrity as a consequence of helminth-induced tissue 

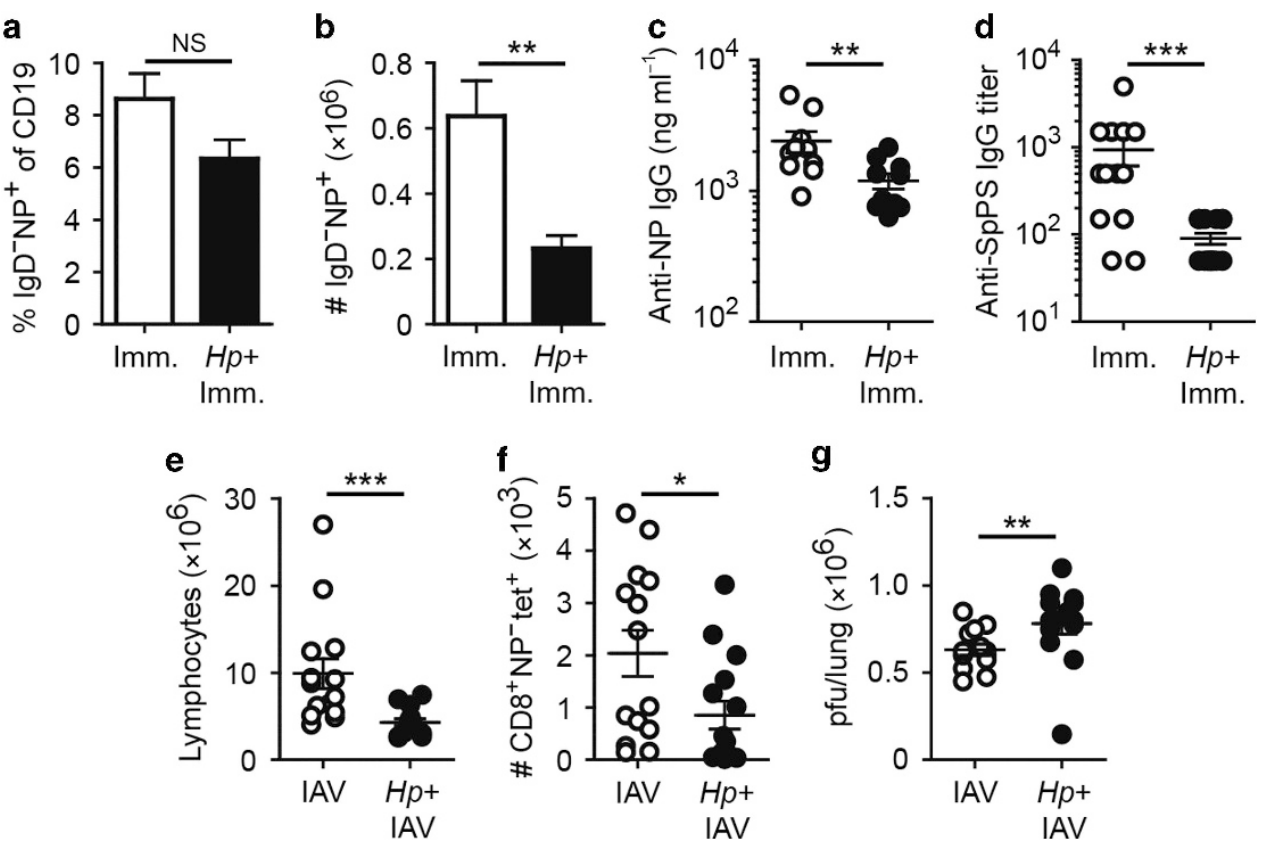

Figure 6 Intestinal helminth infection compromises immunity to heterologous challenge at peripheral sites. C57BL/6 mice were infected with Heligmosomoides polygrus (Hp) or remained naive. Two weeks later, the animals were heterologously challenged. (a-c) One week after footpad immunization with 4-hydroxy-3-nitrophenylacetyl (NP)-KLH in alum, the frequency (a) and number (b) of IgD ${ }^{-}$isotype switched NP-specific B cells in the draining popliteal lymph nodes (LNs) were determined by flow cytometry. (c) Serum anti-NP immunoglobulin G (IgG) titers at day 21 after NP-KLH immunization. (d) $\mathrm{Hp}$-infected or naive mice were immunized intramuscularly with the anti-pneumococcal vaccine formulation Prevnar13 and anti-S. pneumoniae polysaccharide IgG titers were determined 4-5 weeks later. (e, f) Number of total (e) or influenza (influenza A virus (IAV))-specific $\mathrm{CD}^{+}$effector T lymphocytes (f) from the mediastinal LNs of $\mathrm{Hp}$-infected or helminth-naive animals 7 days after intranasal infection with 50 pfu (plaque-forming units) IAV. (g) Pulmonary viral load (pfu) was determined at day 7 post-IAV infection. Data shown are pooled from two independent experiments containing a total of $10-12$ mice per group. Error bars indicate the s.e.m. NS, not significant; ${ }^{\star} P<0.05,{ }^{\star *} P<0.01{ }^{* * *} P<0.001$.

damage. ${ }^{29}$ However, as discussed below, the mechanisms driving naive T-cell accumulation may also contribute to the anti-helminth humoral immune response.

Our results indicated that naive lymphocyte accumulation in the inflamed mesLNs required both $\mathrm{CD} 4{ }^{+}$T-cell activation and LT $\beta R$-dependent signals. Harris and colleagues recently demonstrated that LT $\beta$ production by B cells acts on C-C motif chemokine ligand 19-producing stromal cell populations to reorganize the LN structure, induce LN hyperplasia, and promote $H p$-specific antibody responses. ${ }^{20}$ Importantly, they found IL-4 to promote LT $\beta$ production by B cells in vitro and IL-4 receptor signaling to be critical for mesLN restructuring in vivo. Consistent with these results, we also found that the expression of IL- $4 \mathrm{R} \alpha$ contributes to naive lymphocyte accumulation and LN hyperplasia. Our previous work demonstrated that the dominant source of IL-4 in the reactive mesLNs of $\mathrm{Hp}$-infected mice are $\mathrm{CD} 4{ }^{+} \mathrm{T}$ follicular helper (Tfh) cells. ${ }^{15}$ These studies collectively suggest that, under normal conditions, early IL- 4 secretion by Tfh cells promotes LT $\beta$ production by follicular B cells that expands LT $\beta$ R-expressing stromal cells to reorganize the LN architecture. Indeed, we have found IL- 4 to be produced by CD $4^{+} \mathrm{T}$ cells as early as 5 days post- $\mathrm{Hp}$ infection, a time point that precedes a detectable germinal center B-cell and antibody response. ${ }^{30}$ These results are consistent with another study which also found that LT $\beta$ production by $\mathrm{B}$ cells promotes Th2 responses following $H p$ infection. ${ }^{18}$ However, we also found $\mathrm{B}$ cells to be dispensable for naive T-cell accumulation indicating an additional source of LT $\beta R$ ligand that compensates for B-cell production of LT $\beta$. As our data show that DCs and $\mathrm{CD} 4{ }^{+} \mathrm{T}$ cells express high levels of Tnfsf14 mRNA (the gene encoding for LIGHT, an alternate LT $\beta$ R ligand) compared with B cells and that LIGHT has been previously shown to be important for LN hypertrophy following immunization, ${ }^{19}$ we suggest that DCs and/or T cells can also directly contribute to enhancing naive lymphocyte accumulation in reactive LNs. Based on our results and those of others, we speculate that, following DC-dependent CD4 ${ }^{+}$ $\mathrm{T}$-cell priming, $\mathrm{T}$ cells produce LT $\beta \mathrm{R}$ ligands or elicit production of these factors by DCs facilitating the accumulation of naive lymphocytes in the inflamed mesLNs. Subsequent IL-4 production by developing Tfh cells stimulates B-cell production of LT $\beta$ that along with LIGHT promotes stromal cell activation and mobilization. This intercellular IL-4-LT $\beta$ signaling network then feeds forward to help position $\mathrm{T}$ and $B$ cells to interact in a highly efficient manner to drive the humoral immune response and generate protective antibodies against the intestinal parasite. However, this comes at the cost of compromising immunity to subsequent infection at peripheral sites. Consistent with our studies, deworming individuals prior to bacille Calmette-Guerin vaccination enhances the magnitude of the Mycobacterium tuberculosis-specific T-cell response. ${ }^{31}$ Our results provide one potential explanation for this clinical benefit and may inform vaccination strategies in regions of the world where helminth infections are endemic. 


\section{METHODS}

Animals. BALB/c, 4get, ${ }^{12}$ 4get $\times I l 4 r a \mathrm{KO}$, Tcra KO, JhD, and OVA-TCR transgenic DO11.10 mice all on a BALB/c background and Ifnar $\mathrm{KO}$ and Tnfa $\mathrm{KO}$ mice on a C57BL/6 background were bred and kept under specific pathogen-free conditions and used at 8-12 weeks of age. All experiments were performed under Institutional Animal Care and Use Committee-approved protocols at the Trudeau Institute, Saranac Lake, NY and McGill University, Montreal, Quebec, Canada.

Infections and immunizations. Animals were infected by gavage with 200 third-stage larvae of $\mathrm{Hp}$ as described. ${ }^{10}$ In some experiments, adult $\mathrm{Hp}$ parasites were eliminated by two oral administrations of $100 \mathrm{mg} \mathrm{kg}^{-1}$ pyrantel pamoate (Columbia Laboratories, Ottawa, Ontario, Canada) delivered 2 days apart. For heterologous challenge experiments, some mice were immunized in the footpad with $100 \mu \mathrm{g}$ of NP-KLH (Biosearch Technologies, Novato, CA) precipitated in alum (Imject 77161, ThermoScientific, Waltham, MA) or intramuscularly with $0.5 \mu \mathrm{g}$ Prevnar13 (Pfizer, New York, NY). Influenza infections were performed intranasally with a sublethal dose (50 plaque-forming units) of $\mathrm{H} 1 \mathrm{~N} 1$ strain A/Puerto Rico/8/34 in a $25-\mu$ l volume. Virus was propagated and isolated from Madin-Darby canine kidney (MDCK) cells and titrated with standard plaque assay in MDCK cells. ${ }^{23}$

Antibody and LT $\beta$ R-Ig treatments. At specified time points following $H p$ infection, some mice were intraperitoneally injected with either $100 \mu \mathrm{g}$ anti-CD62L (MEL14) or rat IgG2a isotype control every other day for the indicated period or $150 \mu \mathrm{g}$ LT $\beta \mathrm{R}-\mathrm{Ig}$ or control reagent (provided by Biogen, Cambridge, MA) on days 7 and 10 post- $H p$ infection. ${ }^{32}$ Anti-CD4 (GK1.5, $500 \mu$ g per mouse) or rat IgG2b isotype control was given intraperitoneally 1 day before infection and $\mathrm{CD} 4^{+}$ T-cell depletion after 2 weeks was still $>98 \%$ (as determined by staining for TCR $\beta, \mathrm{CD} 8 \alpha$, and anti-CD4 (RM4-4)).

T-cell transfers. CD4 ${ }^{+} \mathrm{T}$ cells were FACS sorted from the LNs of Thy $1.2^{+}$DO11.10 mice and $5 \times 10^{5}$ cells were transferred intravenously into naive or day $11 \mathrm{Hp}$-infected Thy $1.1^{+}$recipients. One hour later, the number of donor $\mathrm{CD} 4^{+} \mathrm{T}$ cells present in the mesLNs and pooled non-draining inguinal, axillary, and brachial skin-draining LNs was assessed by flow cytometry.

Flow cytometry. Single-cell suspensions were prepared from the mesenteric or non-draining LNs, stained, and analyzed as described. ${ }^{10}$ The following monoclonal antibodies were used for flow cytometry; clone designations are given in parenthesis: CD4 (RM4-5, RM4-4), CD8 $\alpha$ (53-6.7), CD19 (1D3), B220 (RA3-6B2), CD11c (N418), MHCII (M5/114.15.2), CD62L (MEL-14), CD44 (IM7), IgD (11-26c), DO11.10 TCR (KJ1-26), Thy1.1 (OX7), and Thy1.2 (53-2.1) (BD Biosciences, San Jose, CA, eBiosciences, San Diego, CA, or Biolegend, San Diego, CA). NP was conjugated to allophycocyanin as reported ${ }^{33}$ and used in standard antibody staining procedures for antigen-specific $\mathrm{B}$-cell detection. Influenza-specific $\mathrm{CD}{ }^{+}{ }^{+} \mathrm{T}$ cells were identified with NP (ASNENMETM)-loaded $\mathrm{H}_{-} 2 \mathrm{D}^{\mathrm{b}}$ tetramers conjugated to allophycocyanin (1:600 dilution; National Institutes of Health Tetramer Core Facility, Emory University Vaccine Center, Atlanta, GA). Propidium iodide or fixable viability dye (eBiosciences) was used to eliminate dead cells from analysis. Staining for intracellular active Caspase-3 (C92-605) was performed using Cytofix/Cytoperm reagants as suggested by the manufacturer (BD Biosciences). Samples were acquired on a FACS Canto II or LSR Fortessa (BD Biosciences) and analyzed with the FlowJo software (Tree Star, Ashland, OR).

Enzyme-linked immunosorbent assay. Serotype 3 Streptococcus pneumoniae polysaccharide-specific IgG was detected in serum 4-5 weeks following intramuscular challenge by polysaccharide-specific enzyme-linked immunosorbent assay (ELISA). Briefly, 96-well plates were coated with purified polysaccharide (ATCC, Manassas, VA) overnight, washed, and serum dilutions were applied. Horseradish peroxidase anti-mouse IgG detecting antibody (Southern Biotech,
Birmingham, AL) was applied, developed with ABTS buffer, and read on a Molecular Devices (Sunnyvale, CA) SPECTRAmax Plus 384 at $405 \mathrm{~nm}$. NP-specific IgG was detected in serum by heteroclitic NIP (4-hydroxy-5-iodo-3-nitrophenyl)-specific ELISA as described. ${ }^{33}$ Protocol was identical to polysaccharide ELISA except that plates were coated with NIP(11)-OVAL and an IgG anti-NP standard (clone PeVch $\gamma 1 / \lambda 1$, BioXcell, West Lebanon, NH) was used.

Quantitative reverse transcriptase-PCR. mesLN cells were sorted into the populations described in Figure 4 using a FACS Aria III prior to RNA extraction. mRNA was extracted using the Qiagen (Germantown, MD) RNeasy Micro Kit and cDNA samples were prepared as previously described. ${ }^{15}$ Custom primer sequences were generated using the Primer3 software for the following genes: Lta, Fwd: 5'-CAGCAAGCAGAACTCACTGC-3', Rev: 5'-CACTGAGGAGA GGCACATGG- $3^{\prime}$; $L t b$, Fwd: $5^{\prime}$-ACCTCATAGGCGCTTGGATG- $3^{\prime}$, Rev: $5^{\prime}$-ACGCTTCTTCTTGGCTCGC-3'; Tnfsf14, Fwd: 5' -GCATGG AGAGTGTGGTACAGC-3'，Rev: 5'-TGTCTCCAAGACGTTGAT GC-3'; and Hprt, Fwd: 5'-AGGACCTCTCGAAGTGTTGG-3', Rev: $5^{\prime}$-AACTTGCGCTCATCTTAGGC-3'. Quantitative real-time reverse transcriptase-PCR was performed by Sybr green detection using the Bio-Rad CFX96 Real-Time System and software (Hercules, CA). Fold expression was calculated using the $\Delta \Delta^{\mathrm{CT}}$ method and Hprt as a reference gene.

Confocal fluorescence microscopy. Frozen mesLNs cut into $7-\mu \mathrm{m}$ sections were embedded in optimum cutting temperature compound and labeled with anti-B220 (RA3-6B2), anti-GL7 (GL7), and anti-CD4 (RM4-5) from BD Biosciences. Fluorescent images were obtained with a TCS SP5 confocal microscope and 'stitched' high-resolution whole LN confocal images were obtained using the LAS AF 2.2.1 software (Leica, Wetzlar, Germany).

Statistical analysis. GraphPad Prism (version 5, La Jolla, CA) was used for statistical analysis. Data sets were compared by unpaired, two-tailed Student's or Mann-Whitney $t$-test with the exception of Figures $\mathbf{1 c}$ and $\mathbf{4 g}$ in which a one-way analysis of variance was used followed by Tukey's post hoc analysis. Data are represented as mean \pm s.e.m. if not indicated otherwise. NS, not significant; ${ }^{\star} P<0.05$; ${ }^{* *} P<0.01 ;{ }^{* *} P<0.001$

SUPPLEMENTARY MATERIAL is linked to the online version of the paper at http://www.nature.com/m

\section{ACKNOWLEDGMENTS}

We thank Dr Ghislaine Fontés for her technical assistance in the completion of this work. This research was supported by funds from Trudeau Institute, the National Institutes of Health grant Al076479 (to M.M.), McGill University Faculty of Medicine (to I.L.K.), and the Canadian Institutes of Health Research grant 130579. I.L.K. also holds a Canada Research Chair Award.

\section{AUTHOR CONTRIBUTIONS}

I.L.K. designed and performed experiments, analyzed data, and wrote the manuscript. K.M., A.P.M., J.D., P.L., and F.T. performed experiments and analyzed data. J.H.F. provided mice. A.V.T., E.A.L., and M.D. provided reagents, conceptual input and analyzed data. M.M. initiated the project, analyzed data, and wrote the manuscript.

\section{DISCLOSURE}

The authors declared no conflict of interest.

(c) 2017 Society for Mucosal Immunology

\section{REFERENCES}

1. Takada, K. \& Jameson, S.C. Naive T cell homeostasis: from awareness of space to a sense of place. Nat. Rev. Immunol. 9, 823-832 (2009). 
2. Link, A. et al. Fibroblastic reticular cells in lymph nodes regulate the homeostasis of naive T cells. Nat. Immunol. 8, 1255-1265 (2007).

3. Hataye, J., Moon, J.J., Khoruts, A., Reilly, C. \& Jenkins, M.K. Naive and memory CD4 + T cell survival controlled by clonal abundance. Science 312, 114-116 (2006).

4. Angeli, V. et al. B cell-driven lymphangiogenesis in inflamed lymph nodes enhances dendritic cell mobilization. Immunity 24, 203-215 (2006).

5. Yang, C.Y. et al. Trapping of naive lymphocytes triggers rapid growth and remodeling of the fibroblast network in reactive murine lymph nodes. Proc. Natl. Acad. Sci. USA 111, E109-E118 (2014).

6. Soderberg, K.A. et al. Innate control of adaptive immunity via remodeling of lymph node feed arteriole. Proc. Natl. Acad. Sci. USA 102, 16315-16320 (2005).

7. Webster, B. et al. Regulation of lymph node vascular growth by dendritic cells. J. Exp. Med. 203, 1903-1913 (2006).

8. Pham, T.H., Okada, T., Matloubian, M., Lo, C.G. \& Cyster, J.G. S1P1 receptor signaling overrides retention mediated by $G$ alpha i-coupled receptors to promote T cell egress. Immunity 28, 122-133 (2008).

9. Anthony, R.M., Rutitzky, L.I., Urban, J.F. Jr., Stadecker, M.J. \& Gause, W.C. Protective immune mechanisms in helminth infection. Nat. Rev. Immunol. 7, 975-987 (2007).

10. Mohrs, K., Wakil, A.E., Killeen, N., Locksley, R.M. \& Mohrs, M. A two-step process for cytokine production revealed by IL-4 dual-reporter mice. Immunity 23, 419-429 (2005).

11. Urban, J.F. Jr., Katona, I.M., Paul, W.E. \& Finkelman, F.D. Interleukin 4 is important in protective immunity to a gastrointestinal nematode infection in mice. Proc. Natl. Acad. Sci. USA 88, 5513-5517 (1991).

12. Mohrs, M., Shinkai, K., Mohrs, K. \& Locksley, R.M. Analysis of type 2 immunity in vivo with a bicistronic IL-4 reporter. Immunity 15, 303-311 (2001).

13. Perona-Wright, G., Mohrs, K., Mayer, K.D. \& Mohrs, M. Differential regulation of IL-4Ralpha expression by antigen versus cytokine stimulation characterizes Th2 progression in vivo. J. Immunol. 184, 615-623 (2010).

14. Mempel, T.R., Henrickson, S.E. \& Von Andrian, U.H. T-cell priming by dendritic cells in lymph nodes occurs in three distinct phases. Nature 427, 154-159 (2004).

15. King, I.L. \& Mohrs, M. IL-4-producing CD4 + T cells in reactive lymph nodes during helminth infection are T follicular helper cells. J. Exp. Med. 206, 1001-1007 (2009).

16. McLachlan, J.B. et al. Mast cell-derived tumor necrosis factor induces hypertrophy of draining lymph nodes during infection. Nat. Immunol. 4, 1199-1205 (2003).

17. Shiow, L.R. et al. CD69 acts downstream of interferon-alpha/beta to inhibit S1P1 and lymphocyte egress from lymphoid organs. Nature 440, 540-544 (2006).
18. Leon, B. et al. Regulation of $\mathrm{T}(\mathrm{H}) 2$ development by $\mathrm{CXCR} 5$ + dendritic cells and lymphotoxin-expressing B cells. Nat. Immunol. 13, 681-690 (2012).

19. Zhu, M., Yang, Y., Wang, Y., Wang, Z. \& Fu, Y.X. LIGHT regulates inflamed draining lymph node hypertrophy. J. Immunol. 186, 7156-7163 (2011).

20. Dubey, L.K. et al. Lymphotoxin-dependent B Cell-FRC crosstalk promotes de novo follicle formation and antibody production following intestinal helminth infection. Cell Rep. 15, 1527-1541 (2016).

21. Moussion, C. \& Girard, J.P. Dendritic cells control lymphocyte entry to lymph nodes through high endothelial venules. Nature 479, 542-546 (2011).

22. Moon, J.J. et al. Naive CD4(+) Tcell frequency varies for different epitopes and predicts repertoire diversity and response magnitude. Immunity 27 , 203-213 (2007).

23. Coulombe, F. et al. Targeted prostaglandin E2 inhibition enhances antiviral immunity through induction of type 1 interferon and apoptosis in macrophages. Immunity 40, 554-568 (2014).

24. Pearce, E.J. \& MacDonald, A.S. The immunobiology of schistosomiasis. Nat. Rev. Immunol. 2, 499-511 (2002).

25. Osborne, L.C. et al. Coinfection. Virus-helminth coinfection reveals a microbiota-independent mechanism of immunomodulation. Science 345 , 578-582 (2014).

26. Potian, J.A. et al. Preexisting helminth infection induces inhibition of innate pulmonary anti-tuberculosis defense by engaging the IL-4 receptor pathway. J. Exp. Med. 208, 1863-1874 (2011).

27. Salgame, P., Yap, G.S. \& Gause, W.C. Effect of helminth-induced immunity on infections with microbial pathogens. Nat. Immunol. 14, 1118-1126 (2013).

28. Grainger, J.R. et al. Helminth secretions induce de novo T cell Foxp3 expression and regulatory function through the TGF-beta pathway. J. Exp. Med. 207, 2331-2341 (2010).

29. Su, C.W. et al. Duodenal helminth infection alters barrier function of the colonic epithelium via adaptive immune activation. Infect. Immun. 79, 2285-2294 (2011).

30. Meli, A.P. et al. The integrin LFA-1 controls T follicular helper cell generation and maintenance. Immunity 45, 831-846 (2016).

31. Elias, D. et al. Effect of deworming on human $T$ cell responses to mycobacterial antigens in helminth-exposed individuals before and after bacille Calmette-Guerin (BCG) vaccination. Clin. Exp. Immunol. 123, 219-225 (2001).

32. Wang, Y. et al. Lymphotoxin beta receptor signaling in intestinal epithelial cells orchestrates innate immune responses against mucosal bacterial infection. Immunity 32, 403-413 (2010).

33. King, I.L. et al. Invariant natural killer T cells direct B cell responses to cognate lipid antigen in an IL-21-dependent manner. Nat. Immunol. 13, 44-50 (2012). 